\title{
Research on the Transmission Mechanism of Virtual Router
}

\author{
LU Peng \\ Hunan University, Guangzhou City Construction College \\ Information Engineering Institute \\ Guangzhou, Guanggdong, China \\ Email:gbxing@163.com
}

\begin{abstract}
: when multiple virtual routers in identic logic network cohabit a shared router platform of physical network, this paper proposes an Interiorly Fast Forwarding Technique (IFFT) based on study of Open Shortest Past First (OSPF) and investigation in generation of FIB. This technique is proved in which reducing delay is the yardstick of solving fast forwarding among multiple virtual routers. Its purpose is to eliminate the hops of packet forwarding and to advance the speed of packet transmitting. Meanwhile, this technique enhances network robustness by reducing network loss ratio that caused by single point of failure.
\end{abstract}

Key words: computer architecture; network virtualization; virtual router; OSFP; logic network; physical network

\section{INTRODUCTION}

Network virtualization makes multiple logical networks running [1] in the same shared physical platform, and it is independent of each other and not interfere with each other. In order to adapt to the trend of the development of the network, the industry has proposed a series of virtual network and services to support the router virtualization architecture [2-3]. The same router in the physical network can deploy multiple virtual routers according to the needs of users. Thus, it can support a variety of network architecture, meet the requirements of the virtual network, and can effectively use the hardware resources, in line with the design concept of the green router [4].

In the same logical network, the mapping relationship between the virtual router and the physical network is more than one. As shown in Figure 1, the node A and node B map to the same router in the physical network. When the router

\author{
GAN Hong \\ Guangzhou City Construction College; 510925 \\ Information Engineering Institute \\ Guangdong, Guangzhou, China \\ Email:ganhongvip@163.com
}

mapping relationship between the virtual router and physical network in the same logical network is more than one, it is affected by the transmission cost of [5], such as delay, link cost, etc..

In view of the characteristics of the virtual router to share the same physical platform and the open shortest path first protocol (OSPF), a fast switching technique is proposed based on the analysis of the routing table. The technology can effectively reduce the number of hops and improve the transmission speed of the packet. At the same time, it can also reduce the packet loss rate and improve the robustness of the network.

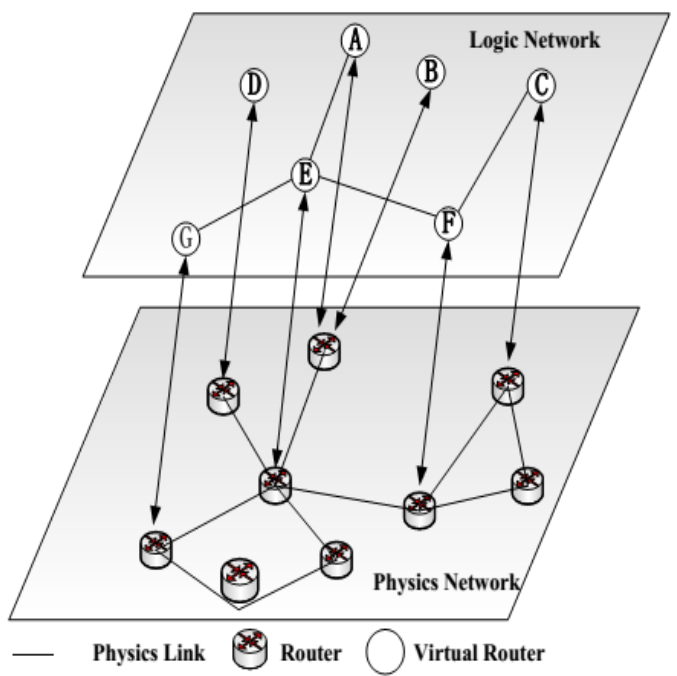

Fig.1: logical network and physical network mapping relationship

\section{IFFT BASIC IDEAS}

In the same logical network, the mapping relationship between the virtual router and the physical network is more than one time. The characteristics of the same physical 
platform are the basis of IFFT. The demand of the link is that the information is not in the same platform, which needs the information of the link, and when the mapping relationship is more than one time, because the virtual router share the same physical platform. In this section, the basic idea of IFFT is explained by comparing the packet forwarding process.

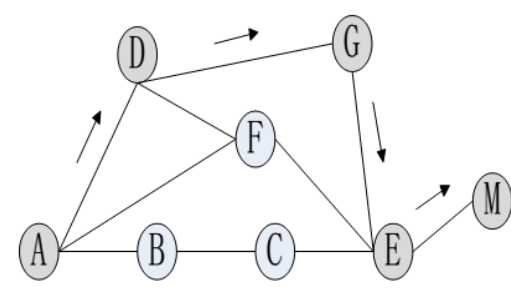

Fig.2: Packet forwarding process

In a logical network, the transmission of a packet is accomplished by a series of routers, such as the storage and forwarding and the link transmission, as shown in Figure 1. Assuming that the packet $\mathrm{N}$ of the network $\mathrm{M}$ in Figure 1 is A-D-G-E-M.

In the same logical network, the virtual router is a multi - to physical network. The virtual router is a shared physical platform, and the system management mechanism of the router in the physical network provides the resources and scheduling, as shown in figure 2. VRA, VRG is the router in the physical network, which is the A and G mapping, VMM is the system management mechanism (Machine Monitor Virtual), which is responsible for resource partitioning, scheduling, etc. RP is a shared physical platform (Physical Real), proposed hardware support. In IFFT, the N passes through the RP to the VRG, and then the VRG check list, the packet is forwarded to the node E, and finally sent to the network $\mathrm{M}$.

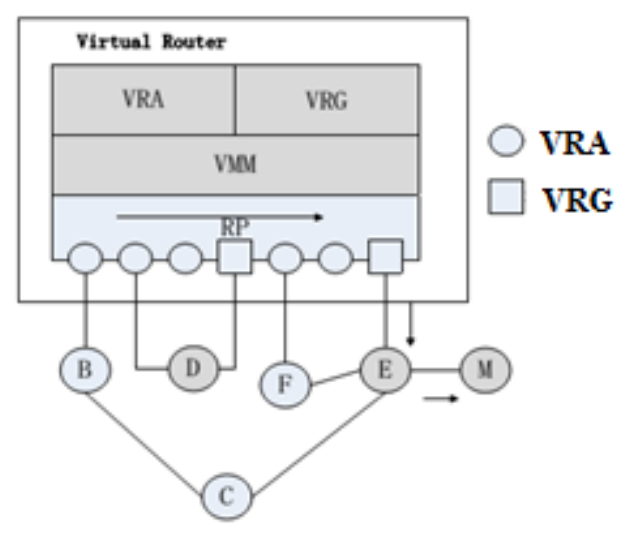

Fig.3: IFFT packet transmission process
Through the comparison of the packet transmission process, IFFT will not need to pass the node $\mathrm{D}$ to the network $\mathrm{M}$ packet. In this way, IFFT reduces the number of hops, and improves the transmission rate of packets. At the same time, when the node $\mathrm{D}$ has a single point of failure, the packets to the network $\mathrm{M}$ are still capable of transmission, reducing the packet loss rate caused by the fault, improve the network's robustness.

\section{IFFT BASIC THEORY}

IFFT is in the same logical network of the virtual router to map the physical network router mapping relationship for a number of time, the transmission mechanism between the virtual router, in fact, the transmission between the virtual router, reducing the number of packets transmitted. At the same time, it can also avoid the partial router single point of failure caused by packet retransmission, etc.. In this section, we can explore the transmission of the message through the theoretical analysis of the virtual router in what case can be achieved using IFFT. In order to be more concise, the focus of IFFT theory is elaborated, and the following two hypotheses are put forward:

The internal gateway protocol of the virtual router in the logical network is the shortest path first protocol OSPF, and mapped to a router in the physical network of multiple virtual routers in the same region of the OSPF division.

Virtual routers have separate routing tables, which do not consider the merging of tables [6-7].

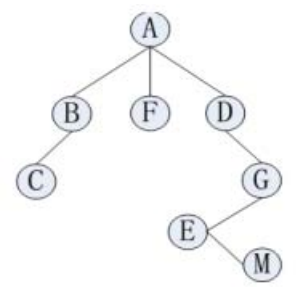

Fig. 4 : SPF spanning tree

In IFFT, the SPF tree is generated by the Shortest First (SPF) algorithm based on the Dijkstra (Path). For example, according to the network topology of Figure 1, the SPF tree is constructed by A as the root node, as shown in Figure 3. Calculate the best path to each network and record the path node information (router ID, port number, load, etc.). If there are nodes in the same physical network, the ID routing table is 
generated, and when the best path is mapped to the nodes of the same physical network, the virtual router ID is mapped to the same physical network as the next hop; otherwise, the next hop is the port number.

In the lookup table, when the next hop of the packet is ID, the packet is copied into the shared memory space by the way of memory copy. The corresponding virtual router will copy the message to its own storage space from the shared memory. Which shared memory uses a pair of one and a directed put-get form. When the message in the VRA is VRG, the process of using the packet to the shared memory space, VRG detects the shared memory space is not empty, the process of the packet is copied to its own space.

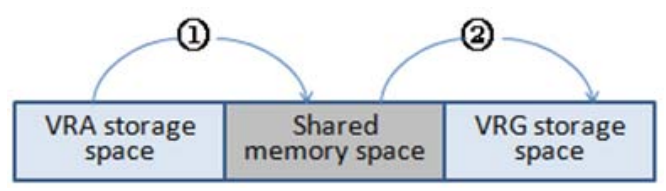

Fig.5 : memory copy mode in virtual router

IFFT is proposed by the theory of acquiring network topology and generating SPF tree in the virtual router, and the best path is not modified to the network. It is only used for the same logical network. Moreover, when the network topology changes, the forwarding table will be modified by the SPF tree to achieve the best path. Therefore, IFFT will not form a route from the ring in the domain.

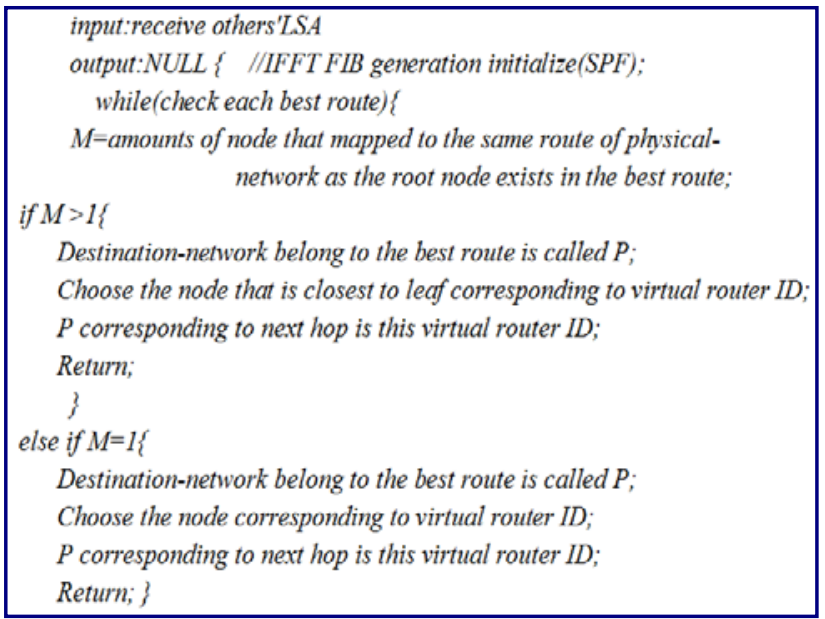

Fig.6: IFFT forwarding table generation algorithm

By the look-up table algorithm description.

\section{IFFT ALGORITHM DESCRIPTION}

IFFT is the way to deal with the next hop in the forwarding table, and the next hop is calculated in the routing table. IFFT has two main routing table generation and routing lookup table. This section describes the routing table generation algorithm and routing lookup table algorithm.

\section{A. IFFT forwarding table generation}

As shown in Fig.6, the generation of IFFT is similar to the existing routing algorithms, but the generation of IFFT is better in the environment of network virtualization. When and only when the network topology changes the virtual router will receive the LSA and check her LSD, which is a virtual router forwarding table to be re generated. The framework of the IFFT forwarding table generation algorithm is shown in fig.6.

\section{B. IFFT routing lookup table}

Routing lookup table is the key to packet forwarding, IFFT has two kinds of packet forwarding, one is directly through the interface of the packet forwarding, and the other is the basic configuration interface.

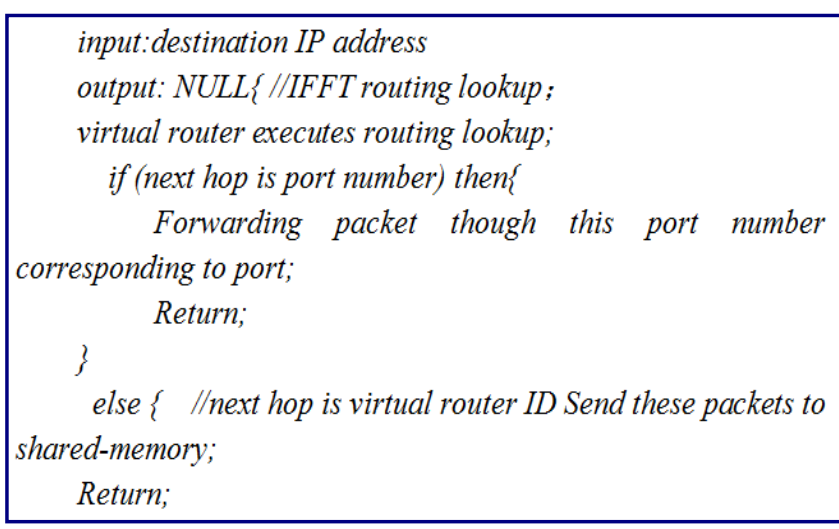

Fig.7 : IFFT lookup table algorithm

\section{PERformance ANALysis}

We use MATLAB to simulate and calculate the packet forwarding rate of different next hop nodes, as shown in figure 8. In Figure 8, the average hop count is 8 when the total number of nodes is 4 and the packet forwarding is 2 . We can find that nodes skip has an effect on the performance of IFFM, so we have a mechanism to enhance the deployment of virtual routers. 


\section{SUMMARY}

The demand of the link is due to the information exchange between the two sides in different platforms, and the same logical network of virtual router mapped to the physical network router mapping relationship for a time, the use of virtual routers to share the same physical platform to achieve information in the internal interface, without the need to link transmission, reduce the number of packets forwarding. IFFT makes full use of the best route to the network in the OSPF protocol to calculate the characteristics of the routing table, by filling in the next hop to determine the packet processing. IFFT can reduce the number of hops, improve the transmission rate. At the same time, it can also reduce the loss caused by single point of failure, improve the network performance.

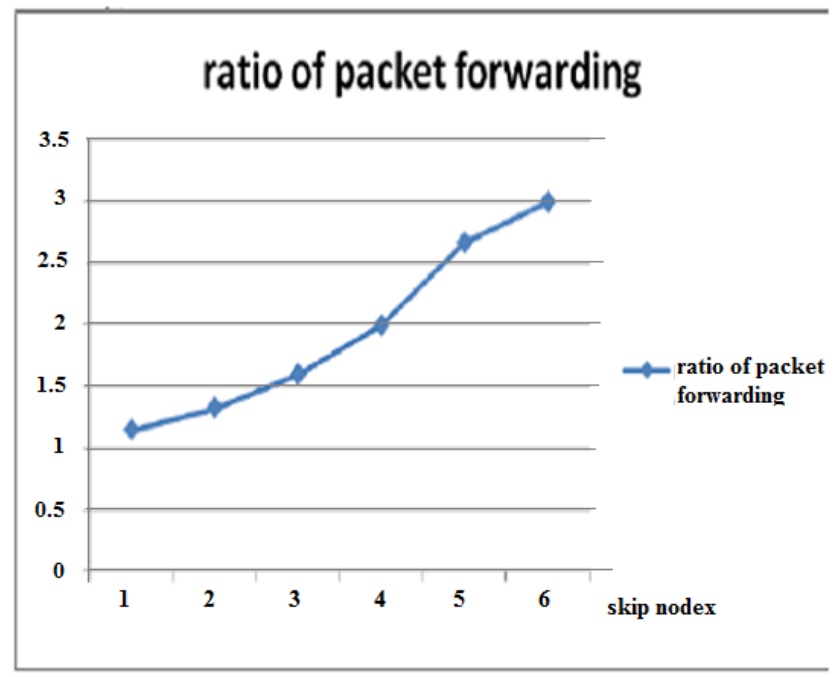

Fig.8: Packet forwarding rate

\section{ACKNOWLEDGMENT}

This work is supported by team research project of Guangdong natural foundation (No. S2012030006242).

\section{REFERENCES}

[1] Zhao Guofeng; Tao Wenping; [J]; virtual technology research of programmable router; Journal of Chongqing University of Posts and Telecommunications (NATURAL SCIENCE EDITION); 2013(01)

[2] the Long Wei; Jia Yin Qi; based on scenario planning the future of Internet applications, classification [J]; Beijing University of Posts and Telecommunications (SOCIAL SCIENCES EDITION); 2013(01)

[3]Grasa E, Junyent G, Figuerola S, et al. UCLPv2: a network virtualization framework built on web services[web services in telecommunications, part II][J].IEEE Communications Magazine, 2008, 46(3):126-134.

[4] Liu Zhongjin; Li Yong; Mao Yang; Su Li; de Peng Jin; Zeng such; based on hardware programming virtual router data plane design and implementation [J]; electronic journal; 2013(07);

[5]J.Fu and J.Rexford, "Efficient IP-Address Lookup with a shared Forwarding Table for Multiple Virtual Routers,"in ACM CoNEXT, 2008.

[6] Mao Yang; Liu Zhongjin; Li Yong; Zeng such; de Peng Jin; Su Li; based on programming the hardware of the virtual router control plane [J]. Journal of Tsinghua University (NATURAL SCIENCE EDITION); 2012(05);

[7] Luo Layong; Hepeng; Hong Tao Guan; Li Zhenyu; Xie Gang;; programming virtual router key technology and prototype system [J]. Journal of computer; 2013(07).

[8]Narvaez P, Siu K Y, Tzeng H Y. New dynamic SPT algorithm based on a ball-and-string model[J]. Networking IEEE/ACM Transactions on, 2001,9(6):706-718.

[9] Zhu Qiusha; the research on the model of data oriented Internet routing architecture based on virtualization technology [D]; Shanghai Jiao Tong University; 2014

[10]Lockwood J W, Mckeown N, Watson G, et al. NetFPGA--An Open Platform for Gigabit-Rate Network Switching and Routing[C]// Proceedings of the 2007 IEEE International Conference on Microelectronic Systems Education IEEE Computer Society, 2007:160-161.

[11]Andersen D, Balakrishnan H, Kaashoek F, et al. Resilient Overlay Networks.[J]. Acm Sigops Operating Systems Review, 2001, 35(5):131-145.

[12]Gupta M, Singh S. Greening of the Internet[C]// In ACM SIGCOMM2003:19-26.

[13]Lyons A M, Neilson D T, Salamon T R, "Energy Efficient Strategies for High Design Telecom Application,"Princeton:Princeton University, 2008. 